\title{
Performance evaluation of LoRa based sensor node and gateway architecture for oil pipeline management
}

\author{
Chavala Lakshmi Narayana, Rajesh Singh, Anita Gehlot \\ School of Electrical and Electronics Engineering, Lovely Professional University, Phagwara, India
}

\begin{abstract}
Article Info
Article history:

Received Jan 17, 2021

Revised Jul 20, 2021

Accepted Aug 9, 2021

\section{Keywords:}

Internet of things

Leaks

Long-range

Pipeline

Wireless sensor network

ABSTRACT

These days, the oil industrial industry is leaning toward employing smart field improvements to streamline various activities in the midstream area. Oil transportation over large distances via pipelines has a cheap cost and high efficiency in this sector. If pipelines are not properly maintained, they may fail, potentially causing catastrophic, long-term, and irreversible consequences on both natural and human conditions. Low power wide area networks (LPWANs) are without a doubt one of the domains that cause the most from industrial fields when it comes to realizing the vision of the internet of things (IoT). Long-range (LoRa) is an emerging LPWAN technology that is particularly useful for transmitting data over long distances. The goal of this work is to offer a methodology for managing oil pipelines over long distances utilizing the LoRa communication protocol and the installation of sensor nodes and LoRa gateways along the pipeline. We also used the optimized network engineering tools (OPNET) simulator to examine various simulation findings of LoRa performance.
\end{abstract}

This is an open access article under the CC BY-SA license.

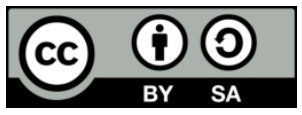

\section{Corresponding Author:}

Chavala Lakshmi Narayana

School of Electrical and Electronics Engineering, Lovely Professional University

Jalandhar-Delhi, Grand Trunk Rd, Phagwara, Punjab 144001, India

Email: laxminarayana0706@gmail.com

\section{INTRODUCTION}

In the oil sector, there are three tiers of activities: upstream, midstream, and downstream. To bring crude oil to the surface, the upstream sector participates in the search for raw materials and exploration from underground and underwater sources [1]. The conveyance of crude oil by pipelines, train, trucks, and other means to processing stations or refineries in downstream sectors is referred to as the middle stream subdivision. The downstream industry plays a significant role in the purification and refining of crude oil. Items made from raw petroleum and combustible gas are marketed and distributed at this stage. Buyers receive goods such as petroleum, kerosene, diesel oil, and a variety of petrochemicals through downstream processes. Attia and Balhassan [2] studied the challenges and advocated that oil flow be carried out smoothly in pipelines. The responsibilities performed by the three subdivisions of the oil industry are depicted in Figure 1. In this article, we look at a few of the major issues that the petroleum industry is facing in the midstream sector, particularly in terms of maintaining a long-distance pipeline. They entail a global network spanning more than 3 million kilometers. Pipeline and hazardous materials safety administration (PHMSA) failure has substantial implications in the United States [3]. According to the facts, there were 432 oil pipeline failures between 2010 and 2015, as indicated in Figure 2. Corrosion, pipe material, and hardware problems are the causes of oil pipeline leaks [4]. Corrosion is a major cause of pipeline failure in oil pipelines. External corrosion, principally galvanic erosion, accounts for approximately $60 \%$ of these, whereas inside corrosion is mostly caused by microbial consumption [4]. Figure 3 depicts the number of barrels of liquid leaked from 1985 to 2015. 


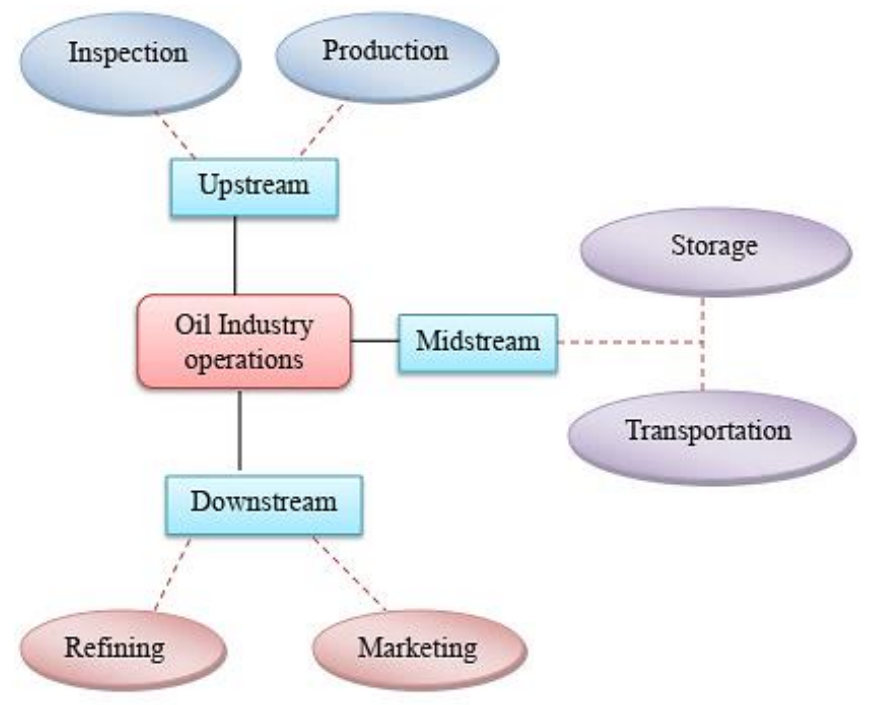

Figure 1. Oil and gas industrial operation sectors

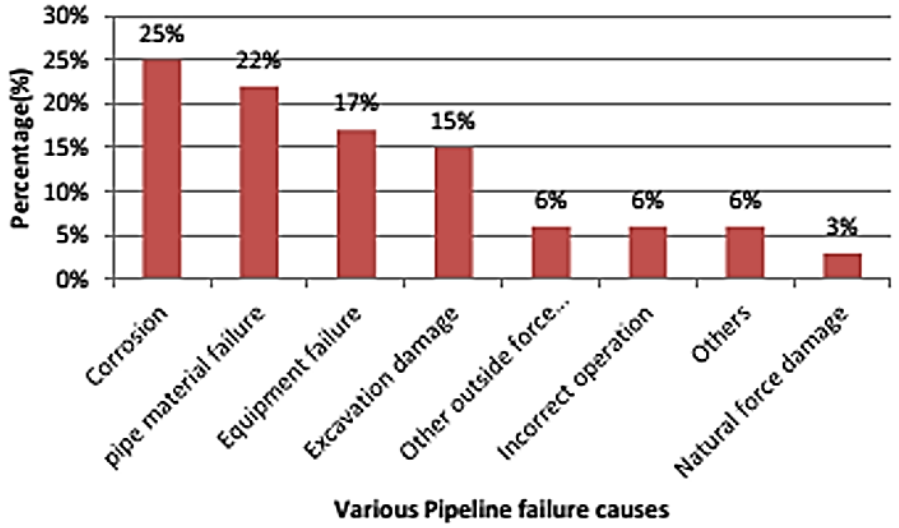

Figure 2. Oil pipeline causes [3]

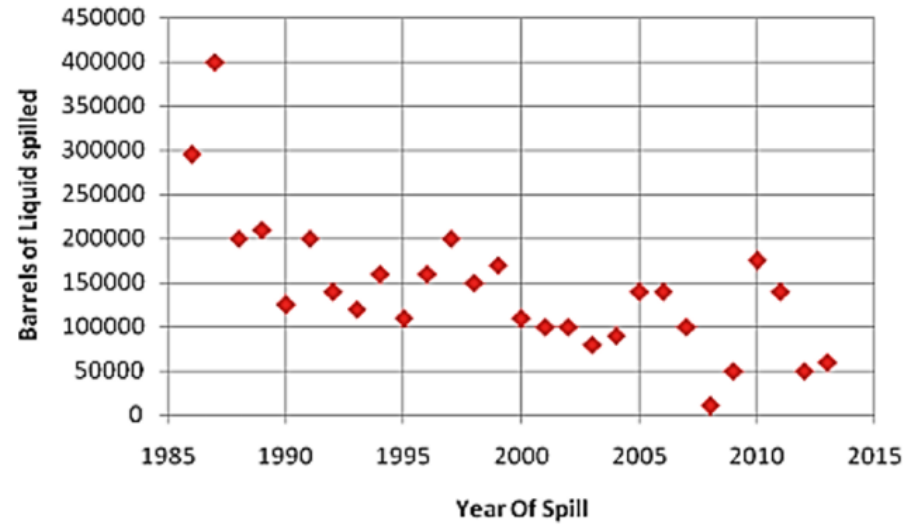

Figure 3. Year wise barrels of liquid spilled [3]

\section{OVERVIEW OF LORa COMMUNICATION TECHNOLOGY}

The long-range (LoRa) is a communication protocol that uses a spread-spectrum technique [5]. This protocol utilizes unlicensed Indian summer monsoon (ISM) groups, i.e., $433 \mathrm{MHz}$ in Asia, $868 \mathrm{MHz}$ in Europe, and $915 \mathrm{MHz}$ in North America. The consequent signal has low noisy levels, engaging high check

Performance evaluation of LoRa based sensor node and gateway... (Chavala Lakshmi Narayana) 
adaptability, and is difficult to stick or recognize [6]. Few works which show highlights of LoRa innovation and detailed data about low power wide area network (LPWAN) innovation and the physical layer (PHY) is portrayed by different parameters like code rate (CR), bandwidth (BW) spreading factor (SF), and transmission power [7]-[10]. Further, messages transmitted using particular spreading components can be gotten simultaneously by long-range (LoRa) base stations [11]. LoRaWAN offers bi-directional availability over multi-kilometers go and with a bit rate between $0.3 \mathrm{kbps}$ to $50 \mathrm{kbps}$. Regardless of whether the correspondence is bi-directional the uplink traffic, from gadget to arrange, is liked [12]. Investigation of the highlights of LoRa innovation and its usage in different application fields has been detailed in various works [13]-[19]. Physical layer measurement is given in reference [20], which includes the bit error rate (BER), signal to noise ratio (SNR), network energy consumption, and received signal strength indicator (RSSI). LoRa uses a "wait and listen" scheme rendering it half-duplex in [21]. Some authors in [22]-[24] proposed a home automation prototype and mobile application framework using LoRa technology. Birajdar et al. [25] proposes building fire detection methodology using LoRa communication protocol. The transmission bandwidth of a technique is called spread spectrum if it is greater than the minimum necessary information transmission bandwidth [26]. Swain et al. [27] development of LoRa tested for agriculture is extracted. Figure 4 shows the basic infrastructure of LoRaWAN.

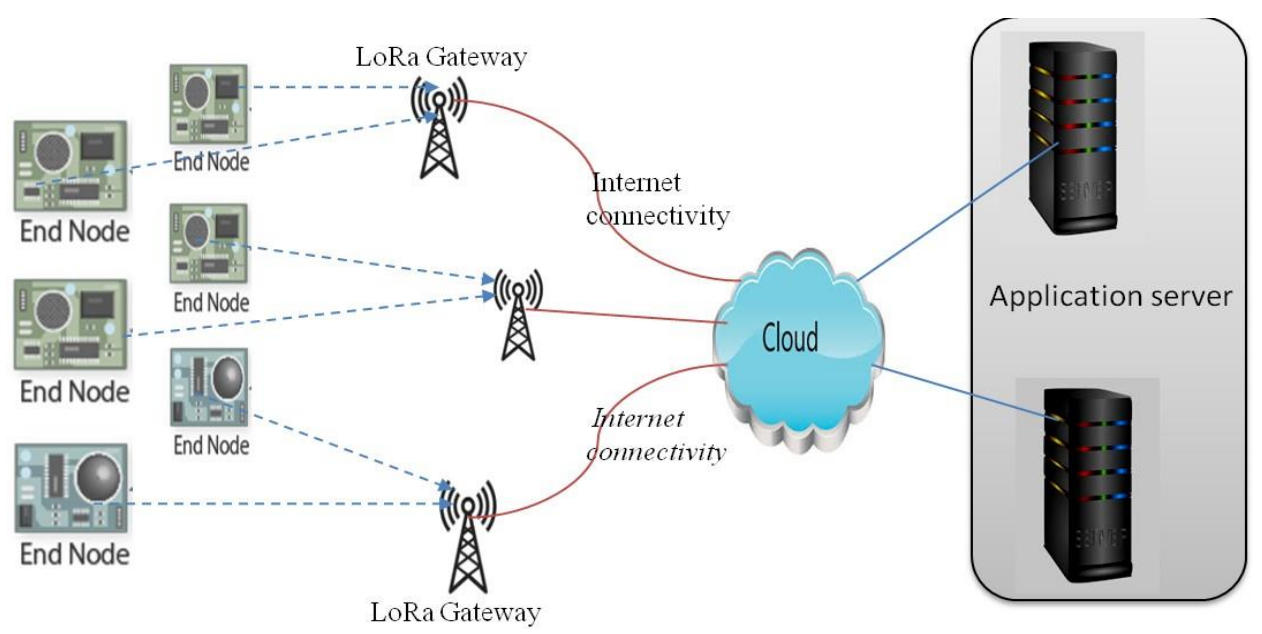

Figure 4. Infrastructure of LoRaWAN

The chirp spread spectrum (CSS) modulation technique is used in LoRa communication. Chirp is a signal that alternates between increasing or decreasing in frequency over time. The number of chips used to display a symbol is determined by the SF value [28]. The relationship between SF and BW is given as (1):

$$
T_{S}=\frac{2^{S F}}{B W}
$$

where $T_{S}$ is the symbol period. The CR carries actual data and ensures that there will be minimal interference during transmission [16]. It is used for forwarding error correction (FEC) and has a range of CR1 to CR4. CR represented as (2),

$$
C R=\frac{4}{4+n}
$$

where $\mathrm{n}$ ranging from $\mathrm{CR} 1$ to $\mathrm{CR}$ 4. Time on air (ToA): The amount of time that passes until a receiver receives a signal is referred to as time on air (ToA). A packet's ToA is measured using a combination of BW, CR, and SF. LoRa packet structure is shown in Figure 5. The length of the preamble is given as (3).

$$
T_{\text {Preamble }}=\left(n_{\text {Preamble }}+4.25\right) T_{\text {Symbol }}
$$

The rate at which bits are transmitted from one place to another is referred to as bit rate or data rate. The data rate of LoRa is represented as (4), 


$$
\mathrm{R}_{\mathrm{b}}=\frac{S F * B W}{2^{S F} * C R}
$$

where $R_{b}$ is nominal bit rate.

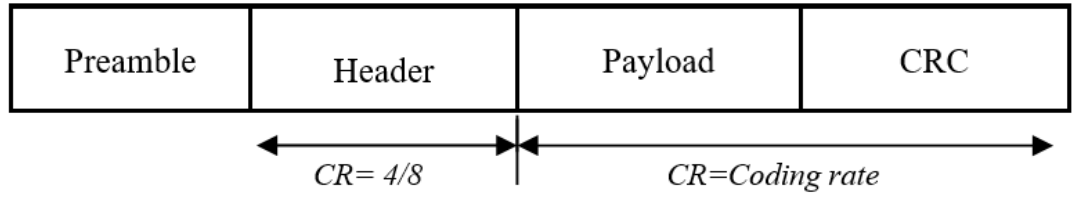

Figure 5. LoRa packet structure

\section{PROPOSED SYSTEM ARCHITECTURE}

In this section, an integrated system for pipeline monitoring is introduced. Figure 6 shows the hybrid architecture of a future oil pipeline monitoring system. Mainly this system includes the pipeline section, monitoring section, and end-user section. Moreover, LoRa communication protocol plays a prominent role in transfer information. Here LoRa is one of the wireless communication protocols which fit to transmit and get information as much as more than 10 kilometers. The primary advantage of this wireless communication protocol is its emphasis on long-range information correspondence with less power utilization. The received information from sensors will be imparted to servers employing LoRa gateway.

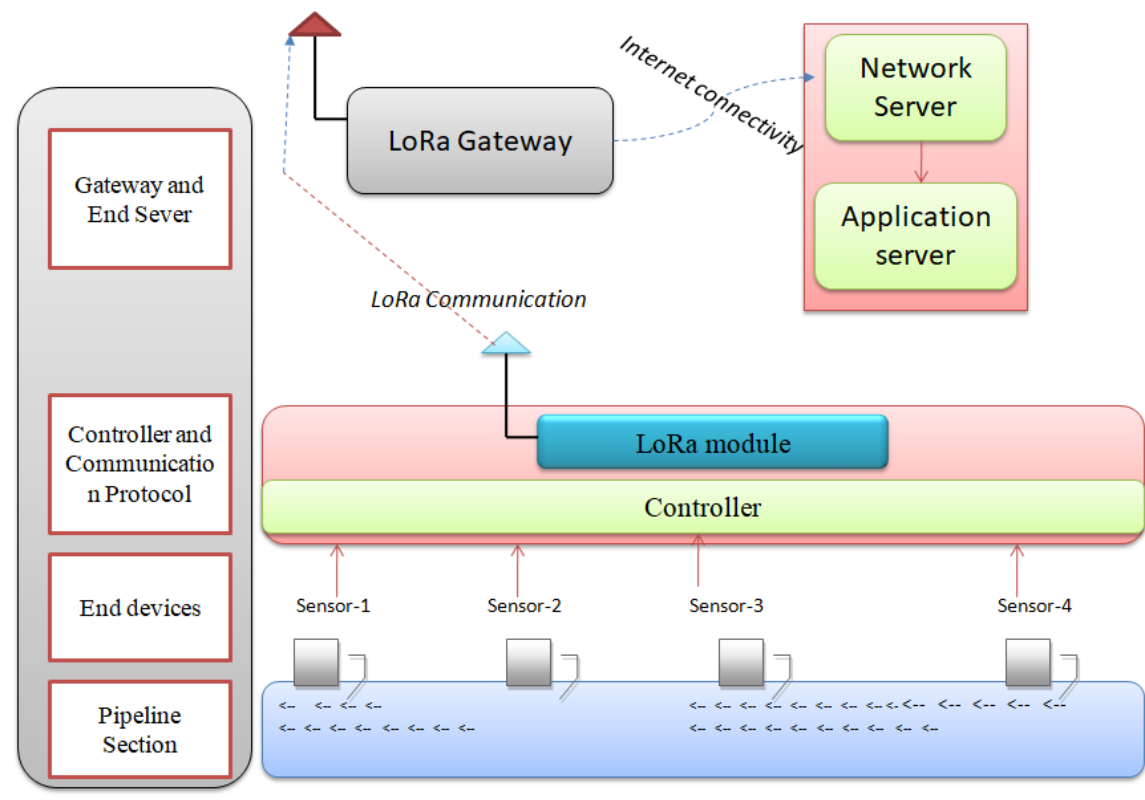

Figure 6. Future oil pipeline monitoring architecture

\subsection{Hardware implementation}

The block diagram for a customized hardware sensor node is shown in Figure 7. A LoRa SX1278 transceiver and an ATmega 328P microcontroller are embedded in the sensor node. The controller is used to obtain sensor data and dissect it, after which the data sent to the wellness activity controller and end-client through LoRa communication protocols.

The Lora based gateway's block diagram, schematic view, prototype, and the deployment of sensor node on the pipeline shown in Figure 8. The gateway, SX1278 LoRa transceiver, and ESP 8266 Wi-Fi module all have an ATmega 328P interface. The gateway aims to send data over multiple communication protocols; in this case, the data received by the SX1278 LoRa transceiver, and the ATmega 328P controller triggers the ESP 8266. The parameters, such as temperature, pressure, humidity, flow rate, and the vibration of the pipeline, are monitored and displayed on the liquid crystal display (LCD). 


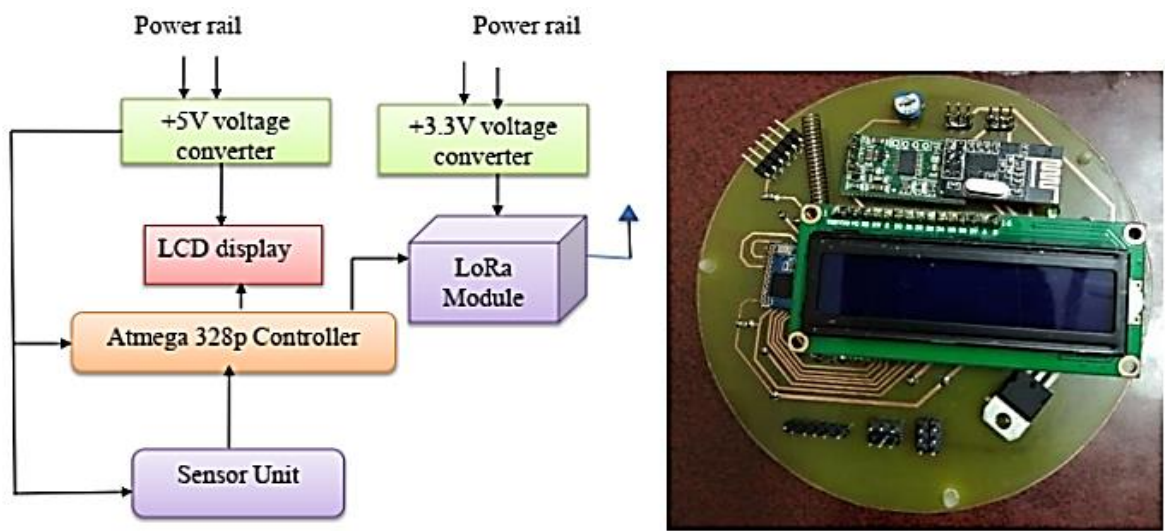

Figure 7. Sensor node
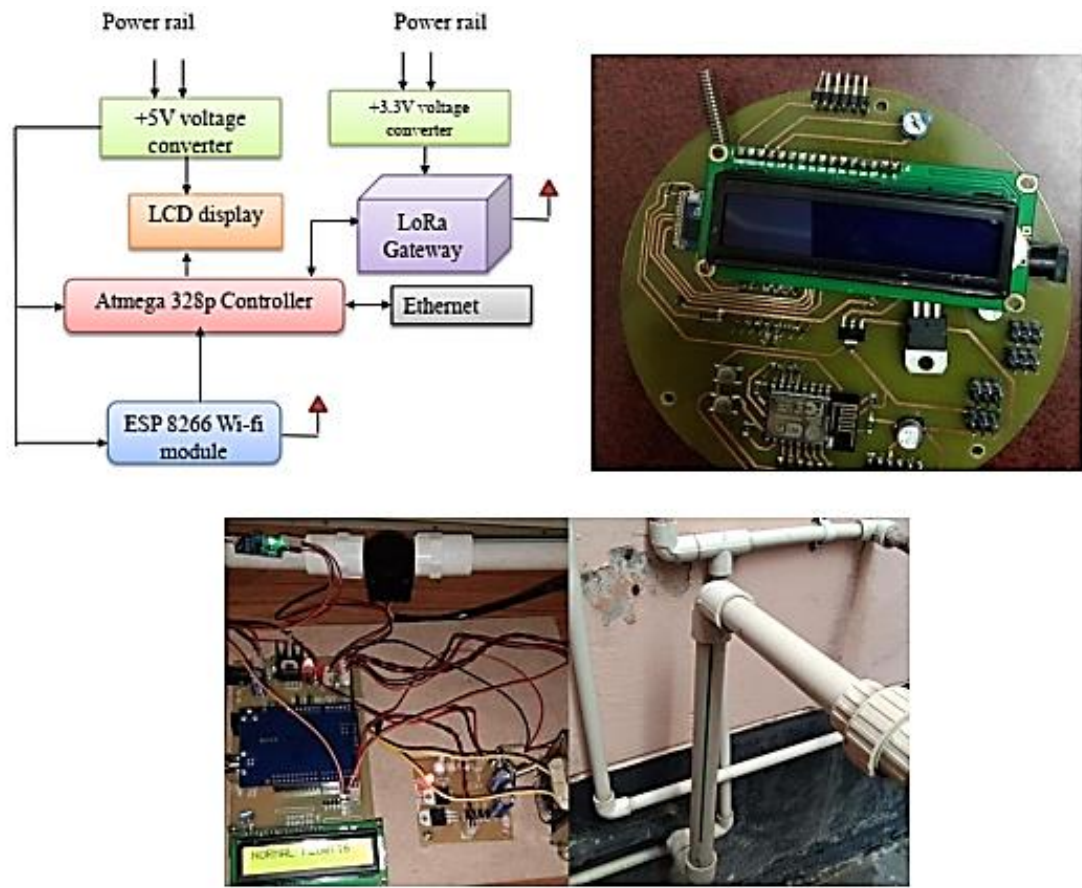

Figure 8. LoRa gateway and deployment of sensor node

\section{PERFORMANCE ANALYSIS}

\subsection{LoRa simulation environment}

The main objectives of this simulation study various quality of service (QoS) boundaries of a network. Here, riverbed modeler variant 17.5 (optimized network engineering tools-OPNET) is utilized for planning and usage of LoRa organization. We have considered a grounds network having a distance of $10 \mathrm{~km}$. The designed network comprises fixed and mobile devices, gateways, cloud, and end servers as shown in the Figure 9. The parameters are as per the following that appeared in the Table 1.

Table 1. LoRa network parameters

\begin{tabular}{cc}
\hline Parameter & Value \\
\hline Spreading factor & SF7 to SF12 \\
Channel bandwidth & $125 \mathrm{KHz}, 250 \mathrm{KHz}, 500 \mathrm{KHz}$ \\
Code rate & $4 / 5$ \\
Channel. Payload & $433 \mathrm{MHz}$, On \\
Start and stop time & Uniform (20, 21), Infinity \\
\hline
\end{tabular}

Int J Elec\& Comp Eng, Vol. 12, No. 1, February 2022: 974-982 


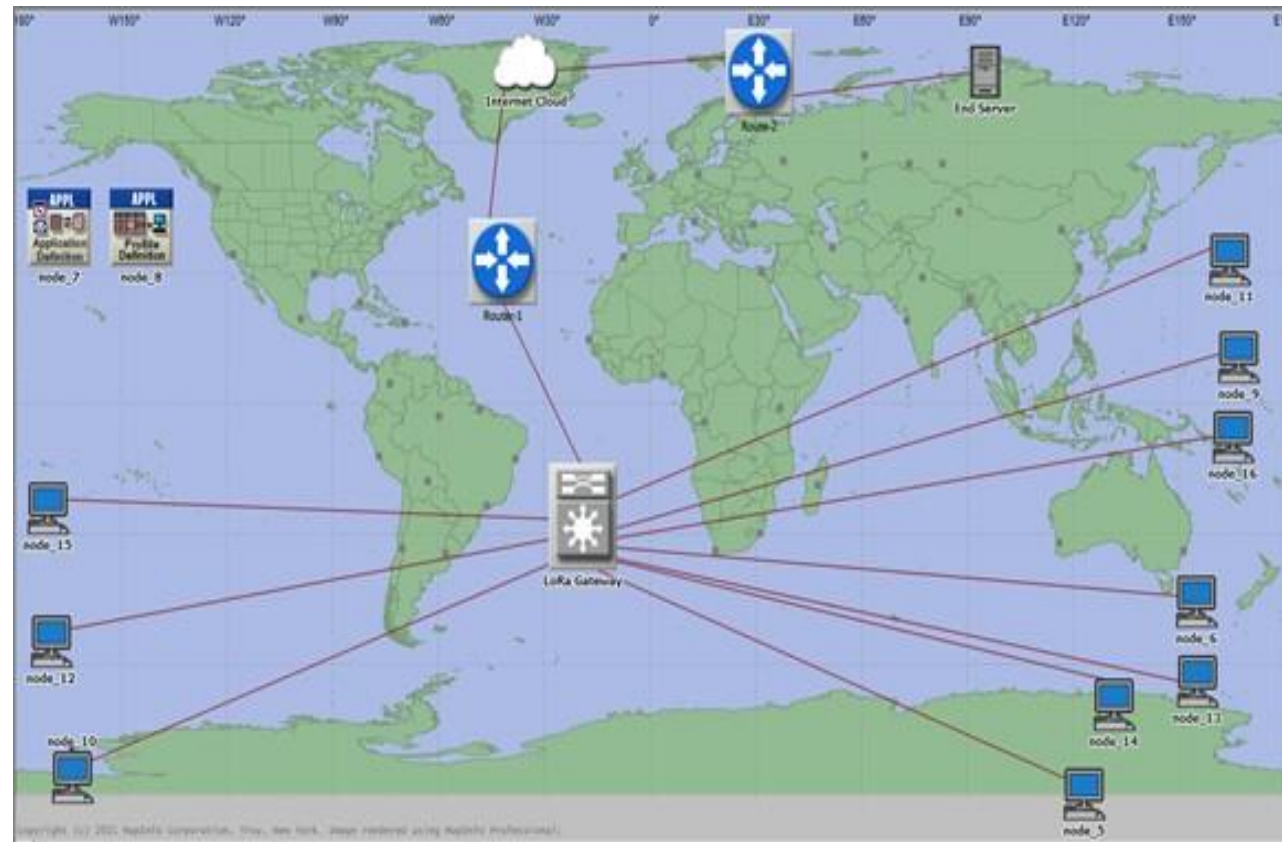

Figure 9. LoRa simulation environment

\subsection{Simulation results}

Figure 10 shows the test results for the received signal strength of the information signals $100 \mathrm{~dB}$ with expanding distance from the gateway. We can conclude that the most extreme correspondence range becomes around $4.8 \mathrm{~km}$ in this environment. Figure 11 shows ToA increases with payload size with various SF. ToA is high with SF-12 400 bytes with 35 bytes of payload and less with SF-7. This implies that the end sensor node consumes more capacity to send information. It is observed that the signal-to-noise ratio (SNR) decreases with distance increases. Up to $3 \mathrm{~km}$, it shows a strong signal and after $3 \mathrm{~km}$ it decreased to below $20 \mathrm{~dB}$. But still gives considerable signal strength as shown in Figure 12.

In Figure 13, simulation results show packet error rate (PER) with various payload sizes. It is observed that slotted Aloha in LoRa with a payload size of 20 bytes for SF-7 and SF-12 PER is almost 5\% and $25 \%$. With the same parameters, it is observed PER is $80 \%$ for SF-12 and $60 \%$ for SF-7 in [14]. Figure 14 presents output power with the LoRaWAN range with various SF. It is seen that, with $S F=7$, the sensor hub needs $25 \mathrm{dBm}$ to communicate information for $0.5 \mathrm{~km}$ with little aggravations. In any case, the sensor needs $10 \mathrm{dBm}$ to send similar data to a similar distance with $\mathrm{SF}=12$.

LoRa sensitivity: The sensitivity of power is usually expressed as a negative number, such as $-130 \mathrm{dBm}$, with a value greater than this indicating decrease insensitivity. The sensitivity from SF 7 to SF 12 is shown in Figure 15. The SF 7 has the maximum sensitivity at BW $10(500 \mathrm{kHz})$, and the SF 12 has the lowest sensitivity at BW $1(8.5 \mathrm{kHz})$. Table 2 illustrates the summarization of simulation results of LoRa performance.

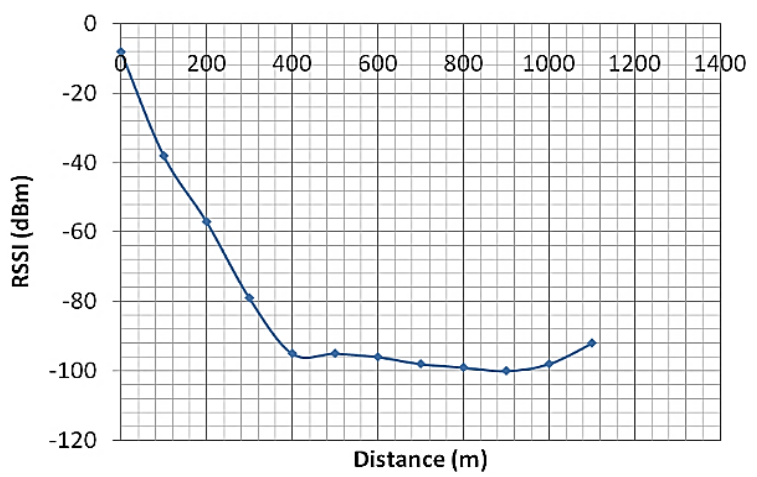

Figure 10. RSSI vs distance (m)

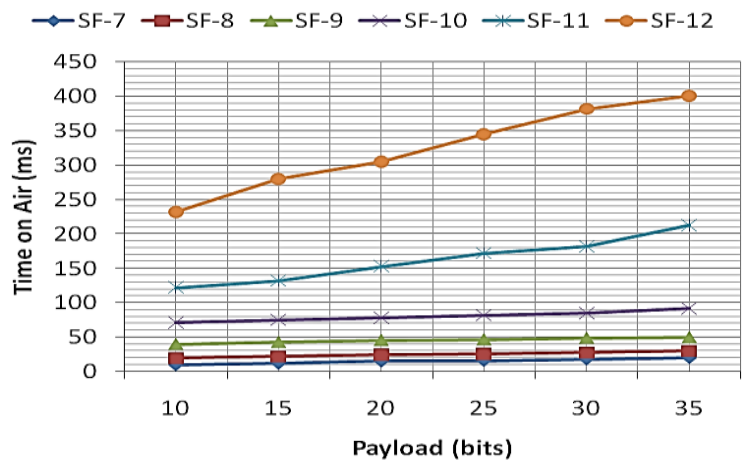

Figure 11. ToA vs payload at different SF 


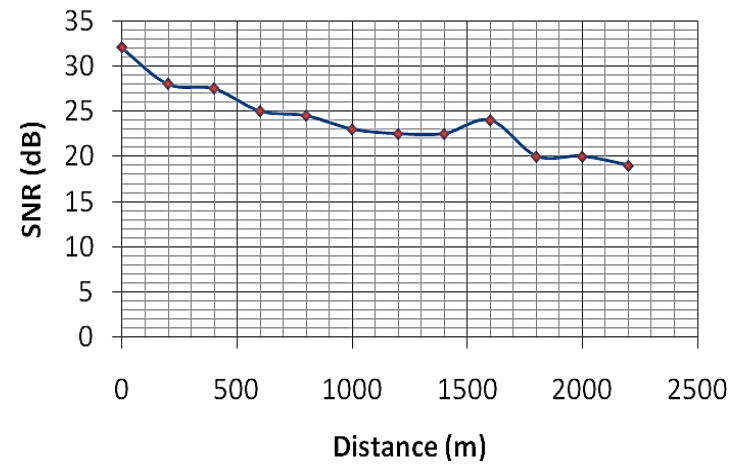

Figure 12. SNR vs distance

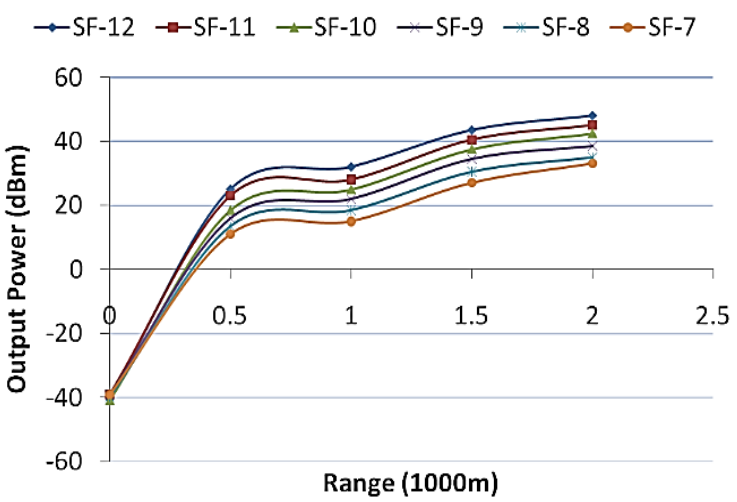

Figure 14. Required output power vs range

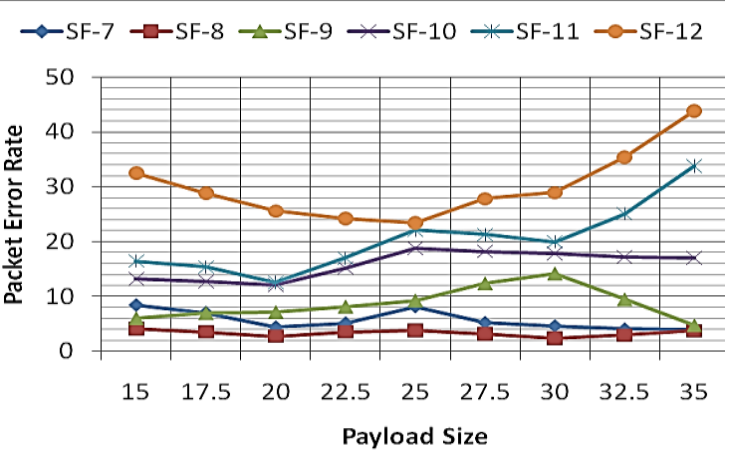

Figure 13. PER vs payload size with various SF

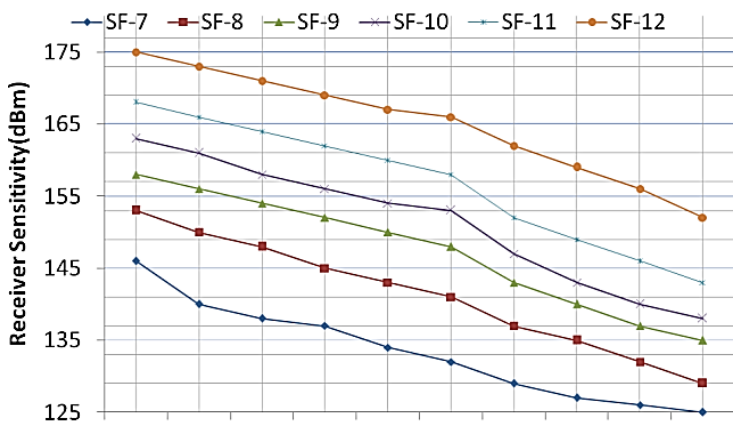

BW 1 BW 2 BW 3 BW 4 BW 5 BW 6 BW 7 BW 8 BW 9 BW 10 Bandwidth (KHz)

Figure 15. LoRa sensitivity

Table 2. Summarization of simulation results

\begin{tabular}{cc}
\hline Characteristic & Specification \\
\hline RSSI & $100 \mathrm{~dB}$ \\
ToA & ToA is good at S-12, 400 ms with 35 bytes of payload \\
SNR & $20-25 \mathrm{~dB}$ \\
Packet Error rate & payload size of 20 bytes for SF-7 and SF-12 PER is almost 5\% and 25\% \\
Output power & Sensor hub needs 10 dBm to communicate information for 0.5 km with SF-12 \\
LoRa Sensitivity & The SF 7 has the highest sensitivity power at 500 kHz.
\end{tabular}

\section{CONCLUSION}

LoRa protocol plays a prominent role in wireless communication and internet of things technology to meet the needs of long-range communication and low power consumption. In this paper, we proposed architecture and implemented hardware LoRa sensor node and gateway customized boards for monitoring various parameters of the oil pipeline management using IoT and Lora WAN. Using the OPNET simulator, we performed simulations on LoRa WAN and evaluated various parameters i.e., SNR, ToA, sensitivity, output power, RSSI, and packet error rate at various spreading factors are plotted.

\section{REFERENCES}

[1] M. Y. Aalsalem, W. Z. Khan, W. Gharibi, M. K. Khan, and Q. Arshad, "Wireless sensor networks in oil and gas industry: Recent advances, taxonomy, requirements, and open challenges," Journal of Network and Computer Applications, vol. 113, pp. 87-89, 2018, doi: 10.1016/j.jnca.2018.04.004.

[2] H. Attia and S. Balhassan, "Design of computerized monitoring and processing system for magnetic field controlling against the phenomenon of black powder in crude oil pipelines," International Journal of Electrical and Computer Engineering (IJECE), vol. 10, no. 5, pp. 4639-4644, doi:10.11591/ijece.v10i5.pp4639-4644.

[3] A. S. Almazyad et al., "A proposed scalable design and simulation of wireless sensor network-based long-distance water pipeline leakage monitoring system," Sensors, 2014, vol. 14, no. 2, pp. 3557-3577, doi: 10.3390/s140203557.

[4] L. Dai, D. Wang, T. Wang, Q. Feng, and X. Yang, "Analysis and comparison of long-distance pipeline failures," Journal of Petroleum Engineering, vol. 2017, Art. no. 3174636, doi: 10.1155/2017/3174636. 
[5] C. Pham, and M. Ehsan, "Dense deployment of LoRa networks: expectations and limits of channel activity detection and capture effect for radio channel access," Sensors, vol. 21, no. 3, Art. no. 825, doi: 10.3390/s21030825.

[6] K. Mekki, E. Bajic, and F. Meyer, "A comparative study of LPWAN technologies for large-scale IoT deployment," ICT Express, vol. 5, no. 1, pp. 1-7, 2019, doi: 10.1016/j.icte.2017.12.005.

[7] A. Augustin, J. Yi, T. Clausen, and W. M. Townsley, "A study of LoRa: Long range \& low power networks for the internet of things," Sensors, vol. 16, no. 9, 2016, Art. no. 1466, doi: 10.3390/s16091466.

[8] S. V. Akram et al., "Performance analysis of iot and long-range radio-based sensor node and gateway architecture for solid waste management," Sensors, vol. 21, no. 8, pp. 2774, 2021, doi: 10.3390/s21082774.

[9] U. Noreen, A. Bounceur, and L. Clavier, "A study of LoRa low power and wide area network technology, "2017 International Conference on Advanced Technologies for Signal and Image Processing (ATSIP), 2017, pp. 1-6, doi: 10.1109/ATSIP.2017.8075570.

[10] A. H. Jebril, A. Sali, A. Ismail, and M. F. A. Rasid, "Overcoming limitations of LoRa physical layer in image transmission," Sensors, vol. 18, no. 10, 2018, Art. no. 3257, doi: 10.3390/s18103257.

[11] Y.-P. E. Wang et al., "A Primer on 3GPP narrowband internet of things," in IEEE Communications Magazine, vol. 55, no. 3, pp. 117-123, March 2017, doi: 10.1109/MCOM.2017.1600510CM.

[12] M. A. Ertürk, M. A. Aydın, M. T. Büyükakkaşlar, and H. Evirgen, "A survey on LoRaWAN architecture, protocol and technologies," Future Internet, vol. 11, no. 10, 2019, Art. no. 216, doi: 10.3390/fi11100216.

[13] J. Petäjäjärvi, K. Mikhaylov, M. Pettissalo, J. Janhunen, and J. Iinatti, "Performance of a low-power wide-area network based on LoRa technology: Doppler robustness, scalability, and coverage," International Journal of Distributed Sensor Networks, vol. 13, no. 3, pp. 1-16, 2017, doi: 10.1177\%2F1550147717699412.

[14] J. Haxhibeqir, F. V. den Abeele, I. Moerman, and J. Hoebeke, "LoRa scalability: a simulation model based on interference measurements," Sensors, vol. 17, no. 6, 2017, Art. no. 1193, doi: 10.3390/s17061193.

[15] F. Adelantado, X. Vilajosana, P. Tuset-Peiro, B. Martinez, J. Melia-Segui, and T. Watteyne, "Understanding the limits of LoRaWAN," IEEE Communications Magazine, vol. 55, no. 9, pp. 34-40, 2017, doi: 10.1109/MCOM.2017.1600613.

[16] M. Bor and U. Roedig, "LoRa transmission parameter selection," In International conference DCOSS, 2017, pp. 27-34, doi: 10.1109/DCOSS.2017.10.

[17] D. Croce, M. Gucciardo, S. Mangione, G. Santaromita, and I. Tinnirello, "Impact of LoRa imperfect orthogonality: Analysis of link-level performance," in IEEE Communications Letters, vol. 22, no. 4, pp. 796-799, Apr. 2018, doi: 10.1109/LCOMM.2018.2797057.

[18] A. Carlsson, L. Kuzminykh, R. Franksson, and A. Liljegren, "Measuring a LoRa network: performance, possibilities and limitations," In International conferenceon Next Generation Wired/Wireless Networking, pp. 116-128, 2018.

[19] E. D. Ayele, C. Hakkenberg, J. P. Meijers, K. Zhang, N. Meratnia, and P. J. M. Havinga, "Performance analysis of LoRa radio for an indoor IoT applications," 2017 International Conference on Internet of Things for the Global Community (IoTGC), 2017, pp. 1-8, doi: 10.1109/IoTGC.2017.8008973.

[20] M. H. Habaebi, I. J. Chowdhury, M. R. Islam, and N. A. B. Zainal, "Effects of shadowing on LoRa LPWAN radio links," International Journal of Electrical and Computer Engineering (IJECE), vol. 7, no. 6, pp. 2970-2976, 2017, doi: 10.11591/ijece.v7i6.pp2970-2976.

[21] O. Kennedy, E. Chukwu, O. Shobayo, E. Noma-Osaghae, I. Okokpujie, and M. Odusami, "Comparativeanalysis of the performance of various active queue management techniques to varying wireless network conditions," International Journal of Electrical and Computer Engineering (IJECE), vol. 9, no. 1, pp. 359-368, 2019, doi:10.11591/ijece.v9i1.pp359-368 .

[22] S. Opipah, H. Qodim, D. Miharja, Sarbini, E. A. Z. Hamidi, and T. Juhana, "Prototype design of smart home system base on LoRa," 2020 6th International Conference on Wireless and Telematics (ICWT), 2020, pp. 1-5, doi: 10.1109/ICWT50448.2020.9243643.

[23] F. A. R. Mu'amar Wildan, E. A. Z. Hamidi and T. Juhana, "The design of application for smart home base on LoRa," 2020 6th International Conference on Wireless and Telematics (ICWT), 2020, pp. 1-6, doi: 10.1109/ICWT50448.2020.9243648.

[24] A. B. Bababe, K. J. Ashish, and K. Rajiv, "LoRa based intelligent home automation system," International Journal of Engineering and Advanced Technology (IJEAT), vol. 6, no. 3, 2017.

[25] G. S. Birajdar, R. Singh, A. Gehlot, and A. K. Thakur "Development in building fire detection and evacuation system-a comprehensive review," International Journal of Electrical and Computer Engineering (IJECE), vol. 10, no. 6, pp. 6644-6654, doi: 10.11591/ijece.v10i6.pp6644-6654.2020.

[26] I. A. Hieder, "Compared to wireless deployment in areas with different environments," International Journal of Electrical and Computer Engineering (IJECE), vol. 9, no. 2, pp. 934-940, 2019

[27] M. Swain, D. Zimon, R. Singh, M. F. Hashmi, M. Rashid, and S. Hakak, "LoRa-LBO: An experimental analysis of lora link budget optimization in custom build iot test bed for agriculture 4.0," Agronomy, vol. 11, no. 5, 2021, doi: 10.3390/agronomy 11050820 .

[28] D. Santos and J. C. Ferreira, "IoT Power Monitoring System for Smart Environments,” Sustainability, vol. 11, 2019, Art. no. 5355, doi: $10.3390 / \mathrm{su} 11195355$.

\section{BIOGRAPHIES OF AUTHORS}

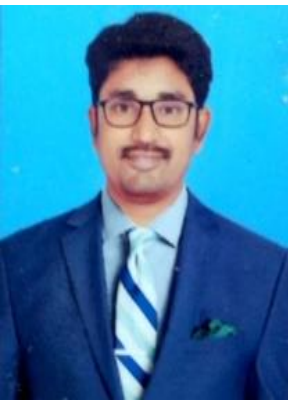

Chavala Lakshmi Narayana (iD) 8 SC P a Research Scholar at Lovely Professional University, Punjab, India. He has completed bachelor's degree in Electronics and Communication Engineering and M.Tech. in Embedded systems. His area of interest is embedded system, internet of things (IoT), wireless sensor networks (WSN). He can be contacted at email: laxminarayana0706@gmail.com. 


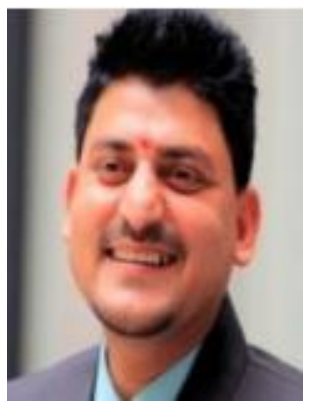

Rajesh Singh (D) SC SC is currently associated with Lovely Professional University as Professor with more than Seventeen years of experience in academics. He has been awarded as gold medallist in M.Tech. from RGPV, Bhopal (M.P) India and honours in his B.E from Dr. B.R. Ambedkar University, Agra (U.P), India. His area of expertise includes embedded systems, robotics, wireless sensor networks, internet of things and machine learning. He has been honoured as keynote speakers and session chair to international/national conferences, faculty development programs, workshops, and webinars. He has Three hundred and ten patents filed including eleven patents grant (8 Australian and 3 Indian patents) and 5 PCT. He has published more than hundred research papers in referred journals/conferences. He has published twentyfour authored and seven edited books in the area of Embedded Systems and Internet of Things with reputed publishers like CRC/Taylor \& Francis, Springer, Narosa, NIPA, River Publishers, Bentham Science, IGI Global, and NOVA Science. He has been featured by Indian and International media for smart systems and devices designed by him including-OBDAS, EParirakshak, Kawach, 20Sec4Life, Ally, Alithis, CT scan Diagnosis, among which five are designed to prevent COVID-19 as per WHO guidelines. He can be contacted at email: srajssssece@gmail.com.

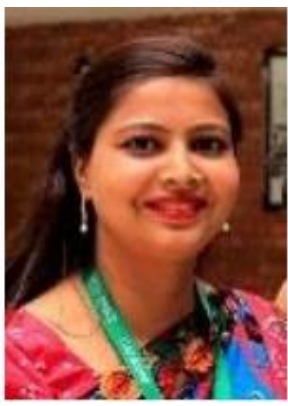

Anita Gehlot (iD 8. SC P is currently associated with Lovely Professional University as Associate Professor with more than Fifteen years of experience in academics. Her area of expertise includes embedded systems, wireless sensor networks and Internet of Things. She has Two hundred and eighty patents filed including eleven patents grant (8 Australian and 3 Indian patents) and 5 PCT. She has published more than Eighty research papers in referred journals/conferences. She has been featured by Indian and International media for smart systems and devices designed by her including-OBDAS, E-Parirakshak, Kawach, 20Sec4Life, Ally, Alithis, CT scan Diagnosis, among which five are designed to prevent COVID-19 as per WHO guidelines. She has published twenty-four authored and six edited books in the area of embedded systems and internet of things with reputed publishers like CRC/Taylor \& Francis, Springer, Narosa, NIPA, River Publishers, Bentham Science, IGI Global, NOVA Science. She can be contacted at email: eranita5@gmail.com. 